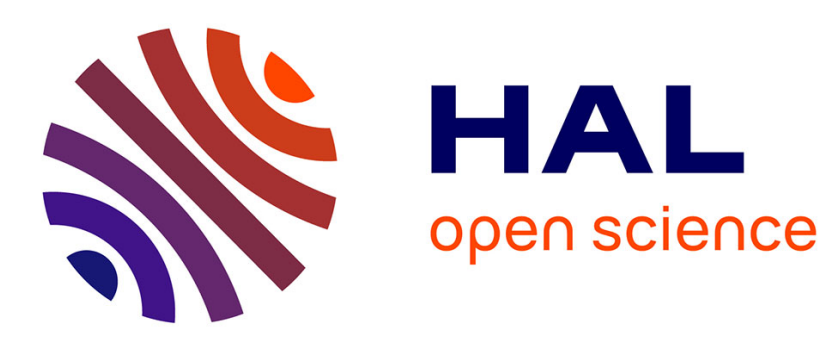

\title{
Primary central nervous system vasculitis: clinical experiences with 21 new European cases
}

\author{
Markus Kraemer, Peter Berlit
}

\section{To cite this version:}

Markus Kraemer, Peter Berlit. Primary central nervous system vasculitis: clinical experiences with 21 new European cases. Rheumatology International, 2009, 31 (4), pp.463-472. 10.1007/s00296-0091312-x . hal-00583534

\section{HAL Id: hal-00583534 https://hal.science/hal-00583534}

Submitted on 6 Apr 2011

HAL is a multi-disciplinary open access archive for the deposit and dissemination of scientific research documents, whether they are published or not. The documents may come from teaching and research institutions in France or abroad, or from public or private research centers.
L'archive ouverte pluridisciplinaire HAL, est destinée au dépôt et à la diffusion de documents scientifiques de niveau recherche, publiés ou non, émanant des établissements d'enseignement et de recherche français ou étrangers, des laboratoires publics ou privés. 
Markus Kraemer, MD; Peter Berlit, MD

Department of Neurology

Alfried Krupp von Bohlen und Halbach Hospital

Alfried-Krupp-Strasse 21

45117 Essen

Germany

Correspondence to:

Dr. med. Markus Kraemer

Department of Neurology

Alfried Krupp von Bohlen und Halbach Hospital

Alfried-Krupp-Strasse 21

45117 Essen

Germany

Tel. +49-201-434-2913

Fax. $+49-201-434-2377$

Email: markus.kraemer1977@gmx.de 


\section{Acknowledgements:}

The authors thank colleagues from the neurological, neurosurgical, neuroradiological and medical departments of Alfried-Krupp-Hospital and colleagues from outside hospitals for good cooperation in management of patients with CNS vasculitis. Especially we want to thank M. W. Baumgaertel, MD (Muenster, Germany), R.R. Diehl, PhD (Essen, Germany), M. Herold, MD (Essen, Germany), A. Kilian (Essen, Germany) and C. Kraemer (Muenster, Germany).

\section{Fundings:}

The study was not supported by funding. 
Original Contribution

Markus Kraemer, MD; P. Berlit, MD

Primary central nervous system vasculitis: Clinical experiences with 21 new European cases 
Background and Purpose: Primary central nervous system vasculitis (PCNSV) represents a rare and poorly-understood form of vascular inflammatory disease restricted to the brain and spinal cord. This study was undertaken to better characterize demographic and clinical features and to evaluate treatments and outcome.

Methods: Our cohort included 21 white patients with PCNSV treated in a single German institution between 2003 and 2008. Clinical and diagnostic features were obtained by retrospective chart review; follow-up information and outcome were obtained prospectively. Clinical findings and outcomes were compared among patients categorized by different characteristics.

Results: The mean age at onset of symptoms was 42.48 years. In 6 patients $(28.6 \%)$ of patients PCNSV diagnosis was established based on positive CNS tissue histopathology. Additional angiography was only performed in 2 of these 6 patients and was negative in both cases. For 13 patients (61.9\%), the diagnosis was confirmed by positive cerebral angiogram. The most frequent clinical presentations over the whole follow up period were hemiparesis $(76.2 \%)$, cerebral ischemia (66.7\%), headache $(57.1 \%)$ and altered cognition (52.4\%). Patients with MRtomographic evident microangiopathy showed significantly worse modified Rankin disability scores during the onset and at last visit compared to the other patients $(p=0.047, p=0.039)$.

Conclusion: Clinical features and course of PCNSV of Europeans analyzed in this German study are comparable to American results but some further reported characteristics of different subtypes could not be duplicated. Although this well defined analysis adds to our knowledge of PCNSV, it also raises important open 
questions. The enigma of PCNSV could only be solved by implementing large prospective multicenter databases and elucidating animal models. 
Primary central nervous system vasculitis (PCNSV) also known as primary or isolated angiitis of the central nervous system is an uncommon disease in which lesions are limited to the brain and spinal cord.[38] It still represents one of the enigmas of clinical neurology[5]. Although it is a rare disease its seriousness makes it important to consider in clinical differential diagnosis. The affliction remains poorly recognized. Since there are no uniform diagnostic criteria, the low specifty and sensifity of the different diagnostic approaches and the scarceness of the disease our existing knowledge is based on incompatible and small case series or single case reports. It is still a major diagnostic and therapeutic challenge and is magnified by numerous unanswered questions regarding epidemiology, predisposition, pathophysiology and outcome[11]. Recently, a working group from the Mayo Clinic, USA, published the retrospective analysis of the largest cohort of such patients ever reported.[11, 28, 35-37, 39, 41] Their analysis included 101 patients with PCNSV seen in Mayo Clinic over two decades from 1983 through 2003[39]. As we frequently are asked to re-evaluate patients with uncommon causes of stroke thought to have inflammatory (PCNSV and secondary caused vasculitides) and non-inflammatory vasculopathies (Moyamoya disease or Sneddon's syndrome), we had the opportunity to include 21 patients with definite PCNSV treated within the short period of 5 years. The design of this study was orientated on the protocols of the most relevant American studies[39] to enable a comparison. 


\section{Material and Methods}

\section{Patient Selection}

We identified all patients diagnosed as "cerebral vasculitis" evaluated at the neurological department of the Alfried Krupp Hospital, Essen, Germany from 2003 through 2008 using our institutional diagnostic linkage system. Inclusion criteria were definite diagnosis of PCNSV. We excluded patients in which vasculitis of the CNS was not definite because other differential diagnoses were discussed. Furthermore we excluded patients who had vasculitis outside the CNS and patients with secondary vasculitis. For cases of uncertain diagnoses we re-evaluated the diagnosis based on the diagnostic criteria proposed by Calabrese and Mallek[10] after reviewing the complete medical records again.

\section{Clinical Data Collection}

In living patients diagnosed with PCNSV $<1$ year before data collection, the asked features were obtained from recent clinical visits. In living patients diagnosed $>1$ year before data collection we tried to obtain follow up information by telephone interview or from outside hospital charts. A standard data collection form was used for the definite cases. Assessment was focussed on demographic, clinical and outcome features. Analysis included demographic data like gender and age at first symptom presentation and the period from symptom onset to diagnosis, clinical presentation at onset of disease and during follow-up period, results from MRI, angiography, CNS biopsy and laboratory investigations. Furthermore treatment data were collected as well as physician's judgment about the response to therapy. Functional status at first visit and latest follow-up was classified by using the modified 
Rankin score (MRS). Angiograms were regarded as highly probable for vasculitis if signs of multiple stenoses or occlusions or areas of vessel-wall segmental thinning or dilatations were observed without evidence for arteriosclerosis or other better explaining conditions. Histopathological marks were considered positive if definite vasculitis was evident, unspecific features like thickened vessel-walls or features of ischemia were judged as vasculitis-negative.

\section{Statistics and Data Analysis}

Statistical comparisons were made using the Mann-Whitney $U$ test (Wilcoxon test) for numeric data. Comparisons of categorical information were done using ChiQuadrate-test or Fisher's exact test when samples were small. All $p$ values were twosided. $\mathrm{P}<0.05$ was considered to be statistical significant. Statistical analysis was completed with SPSS version 17.0.

\section{Results}

\section{Demographic data and diagnosis}

We identified 21 patients treated at Alfried Krupp Hospital, Essen, Germany between 2003 and 2008 regarded as having definite PCNSV. In the preceding process of data collection we excluded 12 uncertain patients because PCNSV was not definite and possible differential diagnoses were considered. The most relevant differential diagnosis was arteriosclerotic vasculopathy. Furthermore we excluded 16 cases of secondary vasculitis.

Table 1 shows the tests used to establish the diagnosis of PCNSV. In 6 patients (28.6\%) of patients PCNSV diagnosis was established based on positive CNS tissue 
histopathology. Additional angiography was only performed in 2 of these 6 patients and was negative in both cases. For 13 patients (61.9\%), the diagnosis was confirmed by positive cerebral angiography. This group of patients diagnosed by angiography included 8 patients who did undergo biopsy. From the 5 angiographypositive patients who underwent biopsy no specimen was positive. In two patients we decided to include them in the analysis despite the angiogram being negative and biopsy was not performed or negative. According to diagnostic criteria of PSNSV formally these were not definite cases of PSNV. However after once again reviewing the medical records and clinical re-evaluation, we were convinced to establish this diagnosis in these two cases and so did not excluded them from data collection in contrast to the other doubtful 12 cases already mentioned.

The mean age of the 21 included PCNSV patients (13 female and 8 male patients) at time of symptom onset was 42.48 years (median, 43, range, 11-65 years). One patient was of Irish, one of Swiss, one of Turkish and 18 patients (85.7\%) of German family origin. The majority of patients $(71.4 \%)$ were older than 37 years at first presentation of the disease. Regarding the time from symptom onset and correct diagnosis, in the majority of patients $(76.2 \%)$ this duration was within 5 months. The median duration from first presentation to correct diagnosis was 2 months with a mean of 18.29 month (range, $0-184$ months). The mean follow up period was 42.6 months (median, 24 months, range, 1-226 months). There was no statistical difference regarding age on first symptom onset, duration to correct diagnosis and follow-up time dividing patients in angiography-positive or biopsy-positive patient and even in patients with microangiopathy or with gadolinium enhancement. In 14 of the PCNSV patients (66.6\%) additional diseases were documented during follow-up. Despite age-related and treatment-related diseases it is notable that in five patients other autoimmune diseases were documented (Hashimoto-thyreoditis in 2, psoriasis 
in 2 and autoimmune hepatitis in 2 patients). Cerebrovascular risk factors were noted in 16 of 21 patients $(76.2 \%)$ which were smoking in 10 patients $(47.6 \%)$ and arterial hypertension in 9 patients (42.9\%). In these patients with cerebrovascular risk factors, this aspect was associated with a higher incidence of ischemic insult over the whole follow-up $(p=0.025)$ and MR-tomographic evident microangiopathy $(p=0.025)$. Furthermore cerebrovascular risk factors were found only in three of six biopsy-proven patients $(p=0.045)$. Patients with cerebrovascular risk factors did not differ in other demographic, clinical, diagnostic or outcome features.

\section{Clinical Symptoms}

The most frequent clinical presentations over the whole follow up period were hemiparesis $(76.2 \%)$, cerebral ischemia $(66.7 \%)$, headache $(57.1 \%)$ and altered cognition (52.4\%). Clinical symptoms at onset of disease were similar to clinical features during follow-up. However headaches seemed to be more frequent at onset of the disease (42.9\% versus $28.6 \%$ ) as well as hemiparesis $(61.9 \%$ versus 38.1$)$. The reverse was true for transient ischemic attacks ( $0 \%$ versus $23.8 \%$ ) and seizures (9.5\% versus 33.3). When patients were divided by diagnostic modality (angiographypositive, biopsy-positive, patients with microangiopathy, patients with gadolinium enhancement), most symptoms appeared with comparable frequency in the different groups (table 2). However, incidence of amnestic syndrome at first presentation was greater in patients with gadolinium enhanced lesions $(p=0.042)$ and gait disorder at the onset seemed to be more frequent $(p=0.056)$ in patients with MRI gadolinium enhancement. Comparing patients with and without microangiopathy those with MRtomographic small vessel involvement showed more often hemiparesis, cerebral ischemia over the whole follow up period ( $p=0.025, p=0.017$, respectively) and more often cognitive decline and cerebral ischemia during follow up $(p=0.024, p=0.032)$. 
Intracranial hemorrhage was very infrequent, it repeatedly occurred in only one biopsy-proven patient. Furthermore none of the patients characterised headache as "thunderclap" headache.

\section{Magnetic resonance findings}

MRI was performed and revealed abnormal findings in all patients (100\%). Ischemic infarctions with diffusion disturbances were seen as the most common feature in MRI in $76.2 \%$ of all patients (50\% in biopsy-positive and $84.6 \%$ in angiography-positive patients). All patients showed bilateral MRI abnormalities. Signs of microangiopathy were also a regularly observed aspect in MRI $66.7 \%$ for all, $66.7 \%$ in biopsy-positive, $61.5 \%$ for angiography-positive patients, respectively). Patients with or without signs of microangiopathy significant differed regarding hemiparesis and ischemic stroke over the whole follow-up and cognitive decline and ischemic stroke in follow-up. Furthermore they presented with a worse MRS. Gadolinium-enhanced lesions were detected in 8 patients (biopsy-positive patients, 66.7\%; angiography-positive patients, 30.8, respectively). Patients with gadolinium enhancement were more often men $(p=0.018)$ and had more frequent amnestic syndromes at disease onset $(p=0.042)$ and a trend to a more frequent symptom onset with gait disturbances $(p=0.056)$ compared with patients without gadolinium enhancing lesions. Patients with gadolinium enhancement were more often treated with cyclophosphamide compared to those without enhancing lesions $(p=0.049)$. MR-angiography (MRA) was performed in 17 of 21 patients and was suggestive for vasculitis in 8 of 17 patients (47.1\%). There was no statistical difference detectable between patients with or without positive MRA regarding demographical, clinical, diagnostic, treatment or outcome features. 


\section{Conventional cerebral angiography}

Conventional cerebral angiography was carried out in 17 of 21 patients $(81.0 \%)$ and was indicative for vasculitis in 13 of 17 patients (76.5\%). Out of the 8 MRangiography-positive patients 6 also showed remarkable conventional angiography findings whereas in 6 angiography-positive patients MR-angiography was normal. Conventional angiography was only performed in 2 of 6 biopsy-approved patients and was normal in both cases. Accordingly, conventional angiography and MRA revealed the same result in 9 of 16 patients (56.2\%) who underwent both investigations. Angiographic-positive and -negative patients did not differ from each other according to demographic data, clinical or diagnostic and outcome features. The association of positive color duplex sonography in angiography-proven patients failed to reach statistical significance $(p=0.063)$.

\section{Histological Features}

CNS biopsy specimens were histological analysed in 12 of 21 patients $(57.1 \%)$. Histopathological examinations revealed definite diagnosis of PCNSV in 6 of 12 patients (50\%). Out of these 6 patients, only in 2 an additional angiogram was performed and both were negative. Patients with positive CNS biopsy differed from the others by a significant lower quantity of abnormal color duplex sonography findings $(p=0.015)$. There were no other statistical differences detectable between biopsy-positive and biopsy-negative patients. Patients who underwent biopsy had a lower incidence of headaches in the whole follow up $(p=0.024)$ and transient ischemic attacks in the follow up $(p=0.08)$ but did not differ in other parameters. 


\section{Laboratory and Cerebrospinal Fluid Studies}

In 19 of 21 patients (90.5\%), CSF examinations were performed and disclosed abnormal findings (cell count $>5$ cells $/ \mu \mathrm{l}$ or total protein concentration $>45 \mathrm{mg} / \mathrm{dl}$ ) in 13 of them (68.4\%) (table 3). Samples were obtained in 12 of 13 angiography-proven (92.3\%) and in 5 of 6 biopsy-proven patients (83.3\%). In both diagnostic groups pathological results of cerebrospinal fluid (CSF) occurred in the similar incidence (60.0\% for biopsy-based and $66.7 \%$ for angiography-based diagnoses, respectively). The typical finding in CSF was a mild lymphocytic pleocytosis combined with an elevated protein level. Cell count was elevated in $68.4 \%$ and total protein was increased in $52.6 \%$ of patients. The mean cell count in PCNSV patients was $34.2 / \mu \mathrm{l}$ (median, $18 / \mu \mathrm{l}$, range, $5-202 / \mu \mathrm{l}$ ), the mean concentration of total protein was 88.4 $\mathrm{mg} / \mathrm{dl}$ (median, $83.1 \mathrm{mg} / \mathrm{dl}$, range 54-142 mg/dl). Oligoclonal banding was found occasionally. In 17 documented data sets with repeated lumbar taps oligoclonal banding was evident at least at one time in 4 patients (23.5\%) with a similar distribution in both diagnostic groups $(25.0 \%$ in biopsy-proven and $27.3 \%$ in angiography-proven cases). In all examinations, there were no hints for alternative diagnoses, particularly infections or neoplastic meningeal infiltrations. Patients with pathological CSF results did not differ from the others in almost all test criteria despite a higher frequency of gait disturbances at symptom onset $(p=0.044)$. Laboratory screening was unremarkable for complement factors C3 and C4, antinuclear antibodies, antibodies to extractable nuclear antigens, antineutrophil cytospasmic antibodies and HIV in all patients, rheumatic factors were mildly elevated in one patient only and special coagulation studies revealed an abnormal result in one patient (heterozygote factor $V$ Leiden mutation). 


\section{Electroencephalopathy and Color Duplex Sonography}

Electroencephalograms were obtained in 15 of 21 patients and showed abnormal findings with dysrhythmias in 4 of them (26.7\%). All patients underwent extra- and transcranial color duplex sonography. Ten of them presented with intracranial vascular abnormalities, 10 with normal intracranial color duplex sonography and only one patient had mild extracranial signs for arteriosclerosis. Out of the 10 patients with pathological color duplex sonography studies, all 9 cases in which cerebral angiography was done were suggestive for vasculitis in angiogram. Positive MRA was associated with positive duplex color duplex sonography in 7 of 9 cases $(77.7 \%)$. Dividing patients in biopsy-approved and angiography-approved diagnostic groups an opposite proportion of color duplex sonography positive patients could be noted (biopsy-based diagnoses, 16.7\%, angiography-approved, 69.2\%). Biopsy-positive patients showed a significant lower fraction of pathological color duplex sonography results compared with the other patients $(p=0.015)$, while higher incidence of positive color duplex sonography in angiography-positive patients failed to reach statistical significance $(p=0.063)$.

\section{Treatments}

Thrombocyte antiaggregation drugs (ASS or clopidrogrel) were prescribed in 12 of 21 patients $(57.1 \%)$. An immunosuppressive therapy was documented in the grand majority of patients (85.7\%). In detail this was intravenous glucocorticoid therapy in $50 \%$, oral glucocorticoid therapy in $77.8 \%$, intravenous and/or oral cyclophosphamide in $38.9 \%$, azathioprine in $27.8 \%$, methotrexate in $22.2 \%$ and mycophenolate mofetile in $22.2 \%$ of immunosuppressive treated patients, respectively. A positive response to therapy was noted in 13 of 18 sufficiently documented patients $(72.2 \%)$. There was 
no statistical significant difference observable in biopsy-approved and angiographyapproved diagnostic groups. Patients with gadolinium enhanced lesions were more often treated with cyclophosphamide $(\mathrm{p}=0.049)$.

\section{Outcome}

Complications of treatments or diagnostic procedures were recognized over the course of the disease in 8 of 20 documented cases (20\%). These were 2 complications after CNS biopsy with cerebral abscess or mild cerebral hemorrhage, respectively. In 6 patients mild complications of treatment like diarrhoea, cough, pneumonia, fever or mild polyneuropathy were observed. Three patients died within documented follow-up, two of them due to progressive cerebral disease, one patient died due to complications associated with the medical therapy. Modified Rankin disability scores were assessed at first presentation and at the last follow-up examination. Median MRS was 2 (range 1-4) at first presentation and 2 (range 1-6) at last visit information. MRS at both times and a functional outcome of 3 or worse was not significant related to different diagnostic groups (angiography-approved, biopsyapproved) or to performance of biopsy, appearance of gadolinium enhanced lesions, pathological CSF results or occurrence of cerebrovascular risk factors. Patients with MR-tomographic evident microangiopathy showed significantly worse MRS at onset and at last visit compared with the other patients $(p=0.047, p=0.039)$. Functional outcome of 3 or worse failed to reach significant difference in patients with microangiopathy compared with the other patients $(p=0.063)$. 


\section{Discussion}

Primary angiitis of the central nervous system (PCNSV) is still one of the enig mas in clinical neurology [5]. It seems to be very rare with an estimated incidence from 2.4 : 1000000 to at less than $1: 2000000[29,39,46]$. Despite the growing knowledge on processes of CNS inflammation, this entity remains poorly understood. Its pathogenesis is still regarded as a mystery, its diagnosis and treatment is still a clinical challenge [46]. Our existing understanding of the disease is based on a number of smaller case series. Since the first histological description in 1922 [5] about 500 cases have been published worldwide [42]. One of the most relevant studies is the recent series of 101 patients from the Mayo Clinic [28, 35-41].

In our study we present data from a cohort of 21 European white patients with PCNSV with details about clinical manifestations, diagnostic and outcome features. This study was intended to discover similarities or differences of our data compared to the previously published cases. Our analysis should enable a better characterization and understanding of this disease [5].

As no reliable prospective data is available our view of the disease is based on retrospective analyses with all possible bias to date. Inclusion and exclusion criteria of the different studies may be a source of inaccuracy by falsely including patients with other differential diagnoses or by falsely excluding PCNSV patients which do not fulfill all diagnostic criteria. The previously suggested diagnostic criteria for PCNSV include symptoms of multifocal or diffuse CNS-disorder with remitting or progressive course, cerebrospinal fluid (CSF) and magnetic resonance imaging (MRI) findings supporting the diagnosis of vasculitis, and finally either an angiography with a vasculitis pattern with multilocular narrowing and vessel occlusions or a leptomenigeal and parenchymatous biopsy proving vasculitis [5, 10, 29]. Diagnostic criteria, sensivity and specifity of angiography and biopsy have to be discussed later. 
However, we have to point out that our analysis not only includes 6 biopsy-approved and 13 angiography-approved patients but also 2 patients with negative or notperformed biopsy and negative angiogram. Inclusion of these 2 cases in contrast to 12 other doubtful cases was decided after detailed re-assessment and review because of clinical confidence of definite PCNSV. So considering of these two cases can be seen as an advantage in the effort to achieve a more representative characterization of the disease.

Nevertheless, in order to avoid misdiagnoses it is important to rule out relevant differential diagnoses based on a sophisticated diagnostic test battery and clinical judgment [12]. Due to our clinical experience the spectrum of differential diagnoses in patients admitted to us for second opinion in evaluation of PCNSV consists of noninflammatory vasculopathy due to arteriosclerosis [18] or drugs [33], Moyamoya disease and syndrome [24] and Sneddon's syndrome [20-22, 25] as well as atypical cases of multiple sclerosis [19]. One other important differential diagnosis of PCNSV is bacterial endocarditis [4], because both conditions may present with similar clinical and auxiliary findings but effort a different treatment regimen [5]. PCNSV should also be distinguished from vasoconstriction syndromes associated with drugs, migraine, hypertension, eclampsia or the post-partum-period [5, 46]. As in spasms after subarachnoid hemorrhage reversible vasoconstriction syndromes (the so-called CallFleming syndrome) often presented with thunderclap headache and are more frequent than PCNSV $[5,11,46]$. As mentioned above we excluded 12 uncertain PCNSV patients in which important differential diagnoses were considered and 16 patients with secondary vasculitis [23]. These cases were associated with systemic disorders like Behcet syndrome [7, 26] (7 cases), Wegener granulomatosis, giant cell arteritis, Churg-Strauss-syndrome [6], sarcoidosis, connective tissue disorders or infectious diseases like borreliosis and neurolues. Unfortunately, we were not able to 
identify the exact number of patients frequently referred to us to re-evaluate suspicion of CNS vasculitis in which this diagnosis was clearly excludable. In the Mayo clinic's cohort this information is also missing [39].

Regarding demographic data and diagnostic test establishing the diagnosis, convincing similarities to Salvarini's cohort are observable [39]. Despite earlier reports of a higher frequency of PCNSV in males, in our cohort female to male ratio was $1.6: 1$ comparable to Salvarini's $1.3: 1$. Diagnostic procedures to establish diagnosis of PCNSV were also utilized as frequent as in the cohort of 101 patients (angiography approved, 61.9\% compared to 69.3\%; biopsy-approved, $28.6 \%$ compared to $30.7 \%$ ). Age distribution also was consistent to former reported ranges. The time from first symptom to correct diagnosis was equally to the Mayo Clinic's data if regarding our median time but longer if regarding the mean time due to 4 patients in which time to diagnoses was much longer than one year [39].

The strength of our demographic data is the accuracy in describing additional diseases and cerebrovascular risk factors. Both are missing in the American paper [39]. In the analysis of 101 patients only one patient with chronic lymphoctic leukemia is noted. In contrast to this Salvarini's working group identified 3 other medical diseases (hypothyreodism and Graves ophthalmopathy) in the analysis of 8 angiography-negative cases[35]. In the subset of 8 PCNSV patients with prominent leptomenigeal enhancement additional diseases were diabetes, hypothyreodism and Crohn's disease [41]. The frequency of co-occurring autoimmune diseases in our study $(23.8 \%)$ points to the clinical problem in districting co-occurrence versus causative systemic disorders. After careful consideration, in individual cases cooccurring autoimmune disorders in patients and their family members should not be regarded as an argument against PCNSV but as a sign for their autoimmune predisposition. In difference to the American working group we assessed 
cerebrovascular risk factors. Despite confidence of diagnosis of definite PCNSV, in these patients with cerebrovascular risk factors this feature was associated with a higher incidence of ischemic lesions over the follow-up period $(p=0.025)$ and MRtomographic evident microangiopathy $(p=0.025)$ without differences in other clinical and outcome features. The occurrence of cerebrovascular risk factors with a great amount of smokers (47.6\%) and patients with arterial hypertension (42.9\%) seemed not to be surprising or unusual in persons over the age of 40 years. Although -agerelated- extracranial signs of arteriosclerosis with mild carotid plaques were recognized in one patient only we want to call attention to the diagnostic problem of differentiating the possibility of co-occurrence of PCNSV with arteriosclerosis from an isolated arteriosclerotic vasculopathy. To the best of our knowledge, this theme is neglected in the existing literature. Nevertheless, in clinical practice, patients with PCNSV should be motivated to avoid smoking and cerebrovascular risk factors should be corrected.

Clinical hallmarks of PCNSV are headaches, strokes, encephalopathy with cognitive disturbances and myelopathy [5]. Headaches are known to occur in two-thirds of patients and are variable in quality and severity [46]. None of our $57.1 \%$ of patients with headache described it as "thunderclap" headache. Non-focal signs, such as neuropsychiatric findings or a decrease in memory [9] are typical for CNS vasculitis. Cognitive alterations were found in similar frequency of patients in our and in the American series [39]. In contrast to our and the Mayo Clinic's statistics a former critical review of PCNSV denied stroke as the presenting symptom in any histological proven case $[39,46]$. We have to discuss that stroke often reveals the disease but perhaps does not represent the retrospective evaluated initial manifestation. In our study only one biopsy-approved patient presented with intracranial hemorrhage, three recurrent hemorrhages appeared in this patient. Summarizing the data of our 
with the Mayo Clinic's cohort we disagree with former reflections that bleedings could be more prevalent than ischemic strokes in PCNSV [46]. Pathophysiologically bleeding is thought to be caused from vessel wall weakness resulting from transmural inflammation $[27,31,46]$.

Magnetic resonance imagings were abnormal in all of our patients and in over $96 \%$ in the Mayo Clinic's study [39]. Normal MR-imaging almost excludes PCNSV. However, the abnormalities observed are absolutely nonspecific [42]. Schmidley pointed out that imaging is nonetheless important, as it might suggest other treatable etiologies for the patient's complaints [42]. Diffuse white matter lesions and microangiopathy were commonly observed. Despite pronunciation of disseminated T2 hypersignals in periventricular white matter in multiple sclerosis, MR-tomographic differentiation to PCNSV can be difficult $[43,46]$. Prominent gadolinium-enhancement of the leptomenix seemed to be less frequent than formerly supposed [13, 31, 46]. Gadolinium-enhanced lesions were observed in $38 \%$ of our patients and in about one third of Salvarini's patients[39]. Regarding the latest widespread usage of MRangiography for evaluation of suspected PCNSV, we want to stress that MRA was suggestive for vasculitis only in $59 \%$ of the American patients and $47.1 \%$ of our patients. Our rate of positive association of MR- and conventional angiography is worse than Salvarini's data $(56.2 \%$ versus $78 \%)$ [39]. So we want to warn to overestimate the sensitivity of MRA which remains unquestionably lower than conventional angiography [46]. Conventional cerebral angiography was indicative for vasculitis in $76.5 \%$ in our patients whereas $90 \%$ of the Mayo Clinic's cohort were positive [39]. In contrast to the former opinion of a more benign course of PCNSV diagnosed on the basis of angiography alone our data does not indicate this comparable with the Mayo Clinic's results [5, 8, 17]. Conventional angiography was only performed in 2 of 6 biopsy-approved patients and was not positive in both cases. 
If the inflammatory findings are limited to arteries less than $500 \mu \mathrm{m}$ in diameter, angiography often is normal [46]. Salvarini et al. observed 8 angiography-negative patients and concluded that this subtype of CNS vasculitis with small vessel involvement beyond resolution of conventional angiography has a more favourable outcome [35]. Furthermore, the American working group described some more characteristics like more common cognitive complaints, higher CSF cell and protein counts and more frequent gadolinium enhancing lesions [35]. In our series we only observed three patients with negative angiogram but could not duplicate the findings of Salvarini and colleagues [35]. The clinically relevant question is if the partly observed differences between the diagnostic groups in Salvarini's and our cohort really represent heterogeneous subtypes of the disease. It is apparent, however, that angiography and biopsy do not provide the same information in all patients [8]. In Salvarini's cohort 18 patients had positive angiograms but negative biopsy findings, in our cohort these were 5 patients [39]. It could be suggested that affected vessels may be larger and were not reached by biopsy. In Yin's Chinese study all cases diagnosed by angiography alone had large vessel involvement [45]. The opposite conclusion can be reached from the fact that in our biopsy-approved patients positive color duplex sonography findings are significantly sparse which also could argue for small vessel involvement. Biopsy-approved PCNSV was found in $50 \%$ of our and $62 \%$ of Salvarini's patients who underwent biopsy. Correct neurosurgical performance of biopsy is crucial for reaching positive results. The ideal diagnostic brain biopsy is a $1 \mathrm{~cm}^{3}$ brain tissue with both gray and white matter as well as leptomeninges and preferably a cortical vessel [29, 42, 46]. The risk of false-negative brain biopsy is not small and can be explained by the segmental involvement of vessels and a possible mismatch between radiological abnormalities and histological predominant lesions $[32,46]$. It is consensus that open biopsy lessens chances of a 
false-negative result compared with stereotactic needle biopsy $[42,46]$. The morbidity rate of brain biopsy $(0.03-2 \%)$ has to be balanced with the risk of unnecessary immunosuppressive treatments $[3,16,42]$. Chu et al. found that morbidity associated with biopsy was less than with "blind" therapeutic immunosuppression [13, 16]. In our cohort those patients which underwent biopsy differed from those without surgery by a lower percentage of headaches in the follow-up period and transient ischemic attacks. This difference obviously is not related to the biopsy procedure but is biased by selection of clinically untypical cases without headaches and transient ischemic attacks to biopsy. Patients with and without surgery do not differ regarding all other parameters. Despite the fact that angiography has become the preferred method of diagnosing PCNSV, we conclude that still biopsy should be regarded as the gold standard [16]. It is important to realize that a negative biopsy certainly does not rule out the condition and it should be considered as an attempt to establish the diagnosis and exclude other conditions [16]. However, it is still a source of controversy what should be done with patients suspected to have a PCNSV having a negative biopsy. One study showed that immunosuppressive treatment is not clearly superior in those patients compared to patients followed up with a wait and see strategy $[1,3,5]$. In contrast to this, other authors conclude that immunosuppressive treatment remains appropriate for those with a consistent clinical and radiological presentation and a negative biopsy [16].

Our laboratory results were mainly within normal ranges as in former studies $[1,10$, 29]. Although a large-scale "laboratory screen" for autoimmune or infectious diseases is essential to exclude treatable differential diagnoses, one should be prepared for occasional "positive" results which are difficult to explain, and may at last be of minor clinical relevance [42]. The lower percentage of performance of lumbar tap in the American study (74.3\% compared with $90.5 \%)$ is perplexing from the European point 
of view. The major importance of CSF examinations also lies in excluding alterative diagnoses [42]. As usually in PCNSV, the typically -but unfortunately- nonspecific finding was mild lymphocytic pleocytosis and elevated protein [32, 42]. In the recent published Chinese series of 8 cases the majority even of biopsy-approved patients presented with normal CSF results $[5,45]$. As mentioned above Salvarini et al. found a significant higher protein level and cell count in CSF in angiography-negative patients which could not be duplicated in our smaller cohort [35]. Unfortunately, in the American study information about oligoclonal banding is absent[39]. In our cohort oligoclonal banding was found occasionally (23.5\%). Both electroencephalography and color duplex sonography are nonspecific. Our result with a significant smaller amount of abnormal intracranial color duplex sonography in biopsy-approved and a trend to more frequent abnormal intracranial duplex sonography in angiographyapproved patients may represent the different involvement of small or large vessels in both diagnostic groups.

No controlled therapeutic trials have been conducted in PCNSV to date $[15,46]$. In an often cited review of 46 patients, 19 of the 20 non-treated patients rapidly progressed either to death or to persistence of severe sequelae $[10,46]$. Treatment regimes are often orientated on experiences in systemic vasculitides. In our cohort an immunosuppressive therapy was given in the grand majority of patients $(85.7 \%)$. In detail this was intravenous glucocorticoid therapy in $50 \%$, oral glucocorticoid therapy in $77.8 \%$, intravenous and/or oral cyclophosphamide in $38.9 \%$, azathioprine in $27.8 \%$, methotrexate in $22.2 \%$ and mycophenolate mofetile in $22.2 \%$ of immunosuppressive treated patients, respectively. The Mayo Clinic's statement that "most patients showed a favourable response to glucocorticoids alone or in combination with cyclophosphamide" is very unprecise $[14,39]$. The experiences on treatments are based on partly inadequately defined populations and on anecdotal 
reports which are impossible to interpret [14]. Combined aggressive therapy should be reserved for those patients with a deteriorating clinical status. Histological proven definite diagnosis should be mandatory in these patients but this once again raises the problem of false-negative biopsy. Alternative treatment regimes for example with intravenous gammaglobulines are not widely used [44]. Furthermore it is often complex to estimate the success of treatments. Clinical assessment of therapy success is often influenced by impression of relapses and remissions. However, this also could be caused by normal recovery of cerebral ischemia [42]. To our experience, the activity of the disease under treatment is appreciable only by combined clinical, CSF and neuroradiological monitoring. Drop of CSF abnormalities is reported corresponds with clinical improvement [32]. The successful use of serial conventionally angiography has been reported [2], but in clinical practise also MRI combined with color duplex sonography studies is increasingly used for follow-up [30, 34]. A prolonged neurological supervision is absolutely necessary. Nevertheless, explicit guidelines for follow-up have not been established to date [11]. So our treatment and outcome data should be interpreted carefully. Clinically and pathophysiologically it makes sense that patients with microangiopathy have a worse outcome. Further interpretations about outcome concluded on the base of the different subgroups of Mayo Clinic's cohort should be regarded considering potential limitations and bias [28, 35-41].

The existing scientific literature on PCNSV is incomplete and difficult to evaluate as much of current understanding comes from smaller case series and single case reports [36].

Our investigation has a number of limitations. Most of the data was assessed retrospective, prospective follow-up was incomplete. It is possible that missing duplication of some characterizations of subgroups described by Salvarini and co- 
workers is caused by smaller study cohort [28, 35-41]. The possibility of referral bias has to be calculated. Sensivity and specifity of different diagnostic approves can influence interpretation of results. What should be done with patients presenting with false-negative biopsy and false-negative angiography but who are clinically considered as definite PCNSV?

Our investigation is elucidating and valuable because it represents one of the largest and most detailed descriptions of European patients with PCNSV.

The strength of our study is the large number of consecutive patients treated over the short period of 5 years. Beside the Mayo Clinic 's cohort with 101 patients our cohort with 21 subjects is large compared with other case series in the existing literature [39]. An important advantage of our short period of 5 years in which we were able to identify 21 patients with PCNSV is - in contrast to the two decades in Mayo Clinic's cohort - the greater change that quality of diagnostic measurements like MRI is equivalent [39]. A further strong point in our analysis is the attempt to measure prospective data. Data collection was focussed on demographic, clinical, diagnostic and outcome data. Study design similar to the Mayo Clinic's cohort makes our investigation comparable [39]. Furthermore this study clearly points out the remaining deficits in knowledge of the disease. To date it is still a challenge to diagnose, treat and follow-up patients thought to have PCNSV. Due to the missing clinical guidelines and the rarity of the disease diagnosis and treatment of PCNSV should be reserve to some clinical centers with great expertise in vasculitis. Furthermore the complex nature of the disease requires an intensive multiprofessional approach with good cooperation of neurological, rheumatologic, neurosurgical and neuroradiological experts in this field. In order to solve some aspects of its enigma, a prospective multicenter collaborative study collecting patients suspected to have cerebral vasculitis as recent demanded by Calabrese, Malloy and Singhal as well as by Berlit 
is urgently needed $[5,11]$. Furthermore the lack of an animal model represents a significant obstacle in achieving new insights in understanding pathophysiological mechanisms in PCNSV [11].

\section{References:}

1. Abu-Shakra M, Khraishi M, Grosman H, Lewtas J, Cividino A, Keystone EC (1994) Primary angiitis of the CNS diagno sed by angiography. Q J Med 87:351-358

2. Alhalabi M, Moore PM (1994) Serial angiography in isolated angiitis of the central nervous system. Neurology 44:1221-1226

3. Alreshaid AA, Powers WJ (2003) Prognosis of patients with suspected primary CNS angiitis and negative brain biopsy. Neurology 61:831-833

4. Berlit P (2009) Isolated angiitis of the CNS and bacterial endocarditis: similarities and differences. J Neurol 256:792-795

5. Berlit P (2009) Primary angiitis of the CNS - an enigma that needs world-wide efforts to be solved. Eur J Neurol 16:10-11

6. Berlit P, Storch B, Schmitt HP (1986) [Diagnosis and therapy of Churg-Strauss allergic granulomatosis]. Eur Arch Psychiatry Neurol Sci $235: 200-205$

7. Berlit P, Stueper B, Fink I, Rebmann V, Hoyer P, Kreuzfelder E, Grosse-Wilde H (2005) Behcet's disease is associated with increased concentrations of antibodies against phosphatidylserine and ribosomal phosphoproteins. Vasa 34:176-180

8. Calabrese LH (1999) Angiographically defined primary angiitis of the CNS: is it really benign? Neurology 52:1302; author reply 1302-1303

9. Calabrese LH, Duna GF, Lie JT (1997) Vasculitis in the central nervous system. Arthritis Rheum 40:1189-1201

10. Calabrese LH, Mallek JA (1988) Primary angiitis of the central nervous system. Report of 8 new cases, review of the literature, and proposal for diagnostic criteria. Medicine (Baltimore) 67:20-39

11. Calabrese LH, Molloy ES, Singhal AB (2007) Primary central nervous system vasculitis: progress and questions. Ann Neurol 62:430-432

12. Ceccarelli A, De Blasi R, Pavone I, Lamberti P, Carella A, Livrea P, Simone IL (2005) Primary angiitis of the central nervous system: a misinterpreted clinical onset of CNS vasculitis. Eur Neurol 53:40-42

13. Chu CT, Gray L, Goldstein LB, Hulette CM (1998) Diagnosis of intracranial vasculitis: a multi-disciplinary approach. J Neuropathol Exp Neurol 57:30-38

14. Elkind MS, Wright C (2008) Cerebral vasculopathy does not equal primary central nervous system vasculitis. Ann Neurol 64:228; author reply 229

15. Fountain NB, Lopes MB (1999) Control of primary angiitis of the CNS associated with cerebral amyloid angiopathy by cyclophosphamide alone. Neurology 52:660-662

16. Goldstein LB (2008) Primary central nervous system vasculitis: is biopsy appropriate? Ann Neurol 64:228; author reply 229

17. Goldstein LB, Chu CT, Gray L, Hulette CM (1999) Angiographically defined primary angiitis of the CNS: is it really benign? Neurology 52:1302; author reply 1302-1303

18. Kis B, Liebig T, Berlit P (2007) Severe supraaortal atherosclerotic disease resembling Takayasu's Arteritis. Vasc Health Risk Manag 3:351-354 
19. Kis B, Rumberg B, Berlit P (2008) Clinical characteristics of patients with late-onset multiple sclerosis. J Neurol 255:697-702

20. Kraemer M, Baumgaertel MW, Berlit P (2007) Miscarriage, peripheral thromboses and aortic aneurysm in antiphospholipid-antibody-negative Sneddon's syndrome. J Neurol 254:1599-1600

21. Kraemer M, Berlit P (2008) Cerebral haemorrhage as the presenting form of Sneddon's syndrome. Clin Neurol Neurosurg 110:533

22. Kraemer M, Berlit P (2008) Long-term course of antiphospholipid-antibody-negative Sneddon's syndrome. Clin Neurol Neurosurg 110:1072

23. Kraemer M, Berlit P (2009) Systemic, secondary and infectious causes for cerebral vasculitis: clinical experience with 16 new European cases. Rheumatol Int

24. Kraemer M, Heienbrok W, Berlit P (2008) Moyamoya disease in Europeans. Stroke 39:3193-3200

25. Kraemer M, Linden D, Berlit $P$ (2005) The spectrum of differential diagnosis in neurological patients with livedo reticularis and livedo racemosa. A literature review. $\mathrm{J}$ Neurol 252:1155-1166

26. Kramer M, Baumgartel MW, Neuen-Jacob E, Berlit P (2008) [Myalgia in NeuroBehcet's disease. Medication side effects or expression of underlying disease]. Z Rheumatol 67:232-236

27. Kristoferitsch W, Jellinger K, Bock F (1984) Cerebral granulomatous angiitis with atypical features. J Neurol $231: 38-42$

28. Miller DV, Salvarani C, Hunder GG, Brown RD, Parisi JE, Christianson TJ, Giannini C (2009) Biopsy findings in primary angiitis of the central nervous system. Am J Surg Pathol 33:35-43

29. Moore PM (1989) Diagnosis and management of isolated angiitis of the central nervous system. Neurology 39:167-173

30. Moritani T, Hiwatashi A, Shrier DA, Wang HZ, Numaguchi Y, Westesson PL (2004) CNS vasculitis and vasculopathy: efficacy and usefulness of diffusion-weighted echoplanar MR imaging. Clin Imaging 28:261-270

31. Negishi C, Sze G (1993) Vasculitis presenting as primary leptomeningeal enhancement with minimal parenchymal findings. AJNR Am J Neuroradiol 14:26-28

32. Oliveira V, Povoa P, Costa A, Ducla-Soares J (1994) Cerebrospinal fluid and therapy of isolated angiitis of the central nervous system. Stroke 25:1693-1695

33. Ringelstein A, Bongs K, Sorge-Hadicke B, Berlit P (2005) [Case of inflammatory vasculopathy and encephalopathy caused by treatment with tacrolimus.]. Nervenarzt 76:475-478

34. Ritter MA, Dziewas R, Papke K, Ludemann P (2002) Follow-up examinations by transcranial Doppler ultrasound in primary angiitis of the central nervous system. Cerebrovasc Dis 14:139-142

35. Salvarani C, Brown RD, Jr., Calamia KT, Christianson TJ, Huston J, 3rd, Mesc hia JF, Giannini C, Miller DV, Hunder GG (2008) Angiography-negative primary central nervous system vasculitis: a syndrome involving small cerebral vessels. Medicine (Baltimore) 87:264-271

36. Salvarani C, Brown RD, Jr., Calamia KT, Christianson TJ, Huston J, 3rd, Meschia JF, Giannini C, Miller DV, Hunder GG (2008) Primary central nervous system vasculitis with prominent leptomeningeal enhancement: a subset with a benign outcome. Arthritis Rheum 58:595-603

37. Salvarani C, Brown RD, Jr., Calamia KT, Christianson TJ, Huston J, 3rd, Meschia JF, Giannini C, Miller DV, Hunder GG (2008) Primary central nervous system vasculitis: comparison of patients with and without cerebral amyloid angiopathy. Rheumatology (Oxford) 47:1671-1677 
38. Salvarani C, Brown RD, Jr., Calamia KT, Christianson TJ, Huston J, 3rd, Meschia JF, Giannini C, Miller DV, Hunder GG (2008) Primary CNS vasculitis with spinal cord involvement. Neurology 70:2394-2400

39. Salvarani C, Brown RD, Jr., Calamia KT, Christianson TJ, Weigand SD, Miller DV, Giannini C, Meschia JF, Huston J, 3rd, Hunder GG (2007) Primary central nervous system vasculitis: analysis of 101 patients. Ann Neurol 62:442-451

40. Salvarani C, Brown RD, Jr., Calamia KT, Huston J, 3rd, Meschia JF, Giannini C, Miller DV, Hunder GG (2008) Efficacy of tumor necrosis factor alpha blockade in primary central nervous system vasculitis resistant to immunosuppressive treatment. Arthritis Rheum 59:291-296

41. Salvarani C, Brown RD, Jr., Huston J, 3rd, Hunder GG (2008) Prominent perivascular enhancement in primary central nervous system vasculitis. Clin Exp Rheumatol 26:S111

42. Schmidley JW (2008) 10 questions on central nervous system vasculitis. Neurologist 14:138-139

43. Scolding N (2007) Can diffusion-weighted imaging improve the diagnosis of CNS vasculitis? Nat Clin Pract Neurol 3:608-609

44. Steinbrecher A, Berlit P (2006) Intravenous immunoglobulin treatment in vasculitis and connective tissue disorders. J Neurol 253 Supp1 5:V39-49

45. Yin Z, Li X, Fang Y, Luo B, Zhang A (2009) Primary angiitis of the central nervous system: report of eight cases from Southern China. Eur J Neurol 16:63-69

46. Zuber M (2008) Isolated angiitis of the central nervous system. In: Caplan LR (ed) Uncommon causes of stroke. Cambridge University Press, Cambridge, pp 1-8

Finding Patients, $\mathbf{n}$

Biopsy positive, angiography negative 2

Biopsy positive, angiography not

4

performed

Angiography positive, biopsy negative 5

Angiography positive, biopsy not

8

performed

Angiography negative, biopsy not

performed

Angiography negative, biopsy negative 1

Table 1. Diagnostic Test Findings 


\begin{tabular}{|c|c|c|c|}
\hline Characteristics & $\begin{array}{l}\text { All patients } \\
(\mathrm{n}=21) \\
\mathrm{n}(\%)\end{array}$ & $\begin{array}{l}\text { Patients } \\
\text { diagnosed by } \\
\text { biopsy } \\
(n=6) \\
n(\%)\end{array}$ & $\begin{array}{l}\text { Patients diagnosed } \\
\text { by angiography } \\
(n=13) \\
n(\%)\end{array}$ \\
\hline Headache & $9(42.9)$ & $1(16.7)$ & $7(53.8)$ \\
\hline Altered cognition & $8(38.1)$ & $2(33.3)$ & $6(46.2)$ \\
\hline Hemiparesis & $13(61.9)$ & $4(66.7)$ & $8(61.5)$ \\
\hline Stroke & $10(47.6)$ & $2(33.3)$ & $6(46.2)$ \\
\hline $\begin{array}{l}\text { Transient ischemic } \\
\text { attack }\end{array}$ & $0(0)$ & $0(0)$ & $0(0)$ \\
\hline Ataxia & $6(28.6)$ & $3(50)$ & $3(23.1)$ \\
\hline Seizure & $2(9.5)$ & $0(0)$ & $2(15.4)$ \\
\hline Visual symptoms & $7(33.3)$ & $4(66.7)$ & $2(15.4)$ \\
\hline $\begin{array}{l}\text { Intracranial } \\
\text { hemorrhage }\end{array}$ & $1(4.8)$ & $1(16.7)$ & $0(0)$ \\
\hline Amnestic & $3(14.3)$ & $2(33.3)$ & $1(7.7)$ \\
\hline syndrome & 7 (33.3) & $3(50)$ & $4(30.8)$ \\
\hline Gait disturbances & $1(4.8)$ & $0(0)$ & $1(7.7)$ \\
\hline Nausea & $3(14.3)$ & $0(0)$ & $2(15.4)$ \\
\hline Vertigo & $2(9.5)$ & $0(0)$ & $1(7.7)$ \\
\hline Aphasia & $5(23.8)$ & $1(16.7)$ & $4(30.8)$ \\
\hline Dysarthia & $4(19.0)$ & $1(16.7)$ & $2(15.4)$ \\
\hline Numbness & $9(42.9)$ & $4(66.7)$ & $5(38.5)$ \\
\hline
\end{tabular}

Table 2. Clinical Manifestations at Presentation 


\begin{tabular}{|c|c|c|c|}
\hline Findings & $\begin{array}{l}\text { All patients } \\
(n=19)\end{array}$ & $\begin{array}{l}\text { Patients } \\
\text { diagnosed by } \\
\text { biopsy }(n=5)\end{array}$ & $\begin{array}{l}\text { Patients } \\
\text { diagnosed by } \\
\text { angiography }(n= \\
12)\end{array}$ \\
\hline $\begin{array}{l}\text { Abnormal findings } \\
>5 \text { cells } / \mu l\end{array}$ & 13 & 2 & 7 \\
\hline $\begin{array}{l}\text { Median leucocyte } \\
\text { count (mean) } \\
\text { (range) }\end{array}$ & $\begin{array}{l}18 \\
(34.18) \\
(5-202)\end{array}$ & $\begin{array}{l}14.5 \\
(14.5) \\
(10-19)\end{array}$ & $\begin{array}{l}18 \\
(20) \\
(5-37)\end{array}$ \\
\hline $\begin{array}{l}\text { Abnormal findings } \\
\text { protein }>45 \mathrm{mg} / \mathrm{dl}\end{array}$ & 8 & 2 & 5 \\
\hline Median protein & & & \\
\hline $\begin{array}{l}\text { concentration } \\
\text { (mean) }\end{array}$ & $\begin{array}{l}83.05 \\
(88.39)\end{array}$ & $\begin{array}{l}114.5 \\
(114.5)\end{array}$ & $\begin{array}{l}65 \\
(69.22)\end{array}$ \\
\hline (range) & $(54-142)$ & $(87-142)$ & $(54-92)$ \\
\hline $\begin{array}{l}\text { Oligoclonal } \\
\text { banding }\end{array}$ & 4 & 1 & 3 \\
\hline
\end{tabular}

Table 3. Cerebrospinal Fluid Findings 